\title{
A note on automorphisms of the infinite-dimensional hypercube graph
}

\author{
Mark Pankov \\ Department of Mathematics and Computer Science \\ University of Warmia and Mazury \\ Olsztyn, Poland \\ pankov@matman.uwm.edu.pl
}

Submitted: Dec 16, 2011; Accepted: Oct 26, 2012; Published: Nov 8, 2012

Mathematics Subject Classifications: 05C63, 20B27

\begin{abstract}
We define the infinite-dimensional hypercube graph $H_{\aleph_{0}}$ as the graph whose vertex set is formed by the so-called singular subsets of $\mathbb{Z} \backslash\{0\}$. This graph is not connected, but it has isomorphic connected components. We show that the restrictions of its automorphisms to the connected components are induced by permutations on $\mathbb{Z} \backslash\{0\}$ preserving the family of singular subsets. As an application, we describe the automorphism group of the connected components.
\end{abstract}

Keywords: infinite-dimensional hypercube graph; graph automorphism; weak wreath product of groups.

\section{Introduction}

By [3], typical graphs have no non-trivial automorphisms. On the other hand, the classical Frucht result [4] states that every abstract group can be realized as the automorphism group of some graph (we refer [2] for more information concerning graph automorphisms). In particular, the Coxeter group of type $\mathrm{B}_{n}=\mathrm{C}_{n}$ (the wreath product $S_{2} 2 S_{n}$ ) is isomorphic to the automorphism group of the $n$-dimensional hypercube graph $H_{n}$.

In this note we consider the infinite-dimensional hypercube graph $H_{\aleph_{0}}$. This is the Cartesian product of infinitely many factors $K_{2}$, but it also can be defined as a graph whose vertex set is formed by the maximal singular subsets of $\mathbb{Z} \backslash\{0\}$ (Section 2). This graph is not connected, but it has isomorphic connected components. We show that the restrictions of its automorphisms to the connected components are induced by permutations on $\mathbb{Z} \backslash\{0\}$ preserving the family of singular subsets (Theorem 2). As a simple consequence, we establish that the automorphism group of each connected component is isomorphic to the 
so-called weak wreath product of $S_{2}$ and $S_{\aleph_{0}}$ (Corollary 5). Since $H_{\aleph_{0}}$ is the Cartesian product of infinitely many factors $K_{2}$, the latter statement can be drawn from some results concerning the automorphism group of Cartesian product of graphs $[5,6]$.

\section{Infinite-dimensional hypercube graph}

A subset $X \subset \mathbb{Z} \backslash\{0\}$ is said to be singular if

$$
i \in X \Longrightarrow-i \notin X \text {. }
$$

For every natural $i$ each maximal singular subset contains precisely one of the numbers $i$ or $-i$; in other words, if $X$ is a maximal singular subset then the same holds for its complement in $\mathbb{Z} \backslash\{0\}$. Two maximal singular subsets $X, Y$ are called adjacent if

$$
|X \backslash Y|=|Y \backslash X|=1 .
$$

In this case, we have

$$
X=(X \cap Y) \cup\{i\} \text { and } Y=(X \cap Y) \cup\{-i\}
$$

for some number $i \in \mathbb{Z} \backslash\{0\}$.

Following Example 2.6 in [7], we say that a permutation $s$ on the set $\mathbb{Z} \backslash\{0\}$ is symplectic if

$$
s(-i)=-s(i) \quad \forall i \in \mathbb{Z} \backslash\{0\} .
$$

A permutation is symplectic if and only if it preserves the family of singular subsets. The group of symplectic permutations is isomorphic to the wreath product $S_{2} \prec S_{\aleph_{0}}$ (we write $S_{\alpha}$ for the group of permutations on a set of cardinality $\alpha$, see Section 5 for the definition of wreath product). The action of this group on the family of maximal singular subsets is transitive.

Denote by $H_{\aleph_{0}}$ the graph whose vertex set is formed by all maximal singular subsets and whose edges are adjacent pairs of such subsets. This graph is not connected. The connected component containing $X \in H_{\aleph_{0}}$ will be denoted by $H(X)$; it consists of all $Y \in H_{\aleph_{0}}$ such that

$$
|X \backslash Y|=|Y \backslash X|<\infty .
$$

Any two connected components $H(X)$ and $H(Y)$ are isomorphic. Indeed, every symplectic permutation $s$ on the set $\mathbb{Z} \backslash\{0\}$ induces an automorphism of $H_{\aleph_{0}}$; this automorphism transfers $H(X)$ to $H(Y)$ if $s(X)=Y$.

It is clear that $H_{\aleph_{0}}$ can be identified with the graph whose vertices are sequences

$$
\left\{a_{n}\right\}_{n \in \mathbb{N}} \text { with } a_{n} \in\{0,1\}
$$

and $\left\{a_{n}\right\}_{n \in \mathbb{N}}$ is adjacent with $\left\{b_{n}\right\}_{n \in \mathbb{N}}$ (connected by an edge) if

$$
\sum_{n \in \mathbb{N}}\left|a_{n}-b_{n}\right|=1
$$

Then one of the connected components is formed by all sequences having a finite number of non-zero elements. 


\section{Automorphisms}

Every automorphism of $H_{\aleph_{0}}$ induced by a symplectic permutation will be called regular. An easy verification shows that distinct symplectic permutations induce distinct regular automorphisms. Therefore, the group of regular automorphisms is isomorphic to $S_{2}$ 乙 $S_{\aleph_{0}}$.

Non-regular automorphisms exist. The following example is a modification of examples given in [1, 8], see also Example 3.14 in [7].

Example 1. Let $A \in H_{\aleph_{0}}$ and $B$ be a vertex of the connected component $H(A)$ distinct from $A$. We take any symplectic permutation $s$ transferring $A$ to $B$. This permutation preserves $H(A)$ and the mapping

$$
f(X):=\left\{\begin{array}{cl}
s(X) & X \in H(A) \\
X & X \in H_{\aleph_{0}} \backslash H(A)
\end{array}\right.
$$

is well-defined. Clearly, $f$ is a non-trivial automorphism of $H_{\aleph_{0}}$. Suppose that this automorphism is regular and $t$ is the associated symplectic permutation. For every $i \in$ $\mathbb{Z} \backslash\{0\}$ there exists a singular subset $N$ such that

$$
X=N \cup\{i\} \text { and } Y=N \cup\{-i\}
$$

are elements of $H_{\aleph_{0}} \backslash H(A)$. Then

$$
t(N)=t(X \cap Y)=t(X) \cap t(Y)=f(X) \cap f(Y)=X \cap Y=N
$$

and

$$
N \cup\{i\}=X=f(X)=t(X)=t(N) \cup\{t(i)\}=N \cup\{t(i)\}
$$

which implies that $t(i)=i$. Thus $t$ is identity which is impossible. So, the automorphism $f$ is non-regular.

Theorem 2. The restriction of every automorphism of $H_{\aleph_{0}}$ to any connected component coincides with the restriction of some regular automorphism to this connected component.

A similar result was obtained in [8] for infinite Johnson graphs. The proof of that result is based on the same idea.

\section{Proof of Theorem 2}

Let $A \in H_{\aleph_{0}}$ and $f$ be the restriction of an automorphism of $H_{\aleph_{0}}$ to the connected component $H(A)$. For every $X \in H_{\aleph_{0}}$ we denote by $X^{\sim}$ the set which contains $X$ and all vertices of $H_{\aleph_{0}}$ adjacent with $X$. It is clear that $X^{\sim}$ is contained in $H(A)$ if $X \in H(A)$.

Lemma 3. For every $X \in H(A)$ there is a symplectic permutation $s_{X}$ such that

$$
f(Y)=s_{X}(Y) \quad \forall Y \in X^{\sim} .
$$

THE ELECTRONic Journal of COMBinatorics 19(4) (2012), \#P23 
Proof. We can assume that $f(X)$ coincides with $X$ (if $f(X) \neq X$ then we take any symplectic permutation $t$ sending $f(X)$ to $X$ and consider $t f)$. In this case, the restriction of $f$ to $X^{\sim}$ is a bijective transformation of $X^{\sim}$.

For every $i \in \mathbb{Z} \backslash\{0\}$ one of the following possibilities is realized:

- $i \notin X$,

- $i \in X$.

Consider the first case. Then $-i \in X$ and there is unique element of $X^{\sim}$ containing $i$, this is

$$
Y=\{i\} \cup(X \backslash\{-i\}) .
$$

Since $\left.f\right|_{X \sim}$ is a transformation of $X^{\sim}, f(Y)$ is adjacent with $X$ and the set $f(Y) \backslash X$ contains only one element. We denote it by $s_{X}(i)$. It is clear that $s_{X}(i) \notin X$.

In the second case, $-i \notin X$ and we define $s_{X}(i)$ as $-s_{X}(-i)$. Since $s_{X}(-i)$ does not belong to $X$, we have $s_{X}(i) \in X$.

So, $s_{X}$ is a symplectic permutation on $\mathbb{Z} \backslash\{0\}$ such that

$$
s_{X}(X)=X \text {. }
$$

Now, we check (1).

Let $Y \in X^{\sim}$. Then we have (2) for some $i$ and

$$
s_{X}(Y)=\left\{s_{X}(i)\right\} \cup\left(s_{X}(X) \backslash\left\{-s_{X}(i)\right\}\right)=\left\{s_{X}(i)\right\} \cup\left(X \backslash\left\{-s_{X}(i)\right\}\right)
$$

is the unique element of $X^{\sim}$ containing $s_{X}(i)$. On the other hand, $s_{X}(i)$ belongs to $f(Y)$ by the definition of $s_{X}$. Therefore, $f(Y)$ coincides with $s_{X}(Y)$.

Lemma 4. If $X, Y \in H(A)$ are adjacent then $s_{X}=s_{Y}$.

Proof. Since $X, Y$ are adjacent, we have

$$
X=\{i\} \cup(X \cap Y) \text { and } Y=\{-i\} \cup(X \cap Y)
$$

for some $i \in X$. We can assume that

$$
f(X)=X \text { and } f(Y)=Y .
$$

Indeed, in the general case

$$
f(X)=\{j\} \cup(f(X) \cap f(Y)) \text { and } f(Y)=\{-j\} \cup(f(X) \cap f(Y))
$$

(since $f(X)$ and $f(Y)$ are adjacent); we take any symplectic permutation $t$ sending $j$ and $f(X) \cap f(Y)$ to $i$ and $X \cap Y$ (respectively) and consider $t f$.

Then

$$
s_{X}(X \cap Y)=s_{X}(X) \cap s_{X}(Y)=f(X) \cap f(Y)=X \cap Y
$$


similarly,

$$
s_{Y}(X \cap Y)=X \cap Y
$$

We have

$$
(X \cap Y) \cup\{i\}=X=f(X)=s_{X}(X)=s_{X}((X \cap Y) \cup\{i\})=(X \cap Y) \cup\left\{s_{X}(i)\right\}
$$

and the same arguments show that

$$
(X \cap Y) \cup\{i\}=(X \cap Y) \cup\left\{s_{Y}(i)\right\} .
$$

Therefore,

$$
s_{X}(i)=s_{Y}(i)=i \text { and } s_{X}(-i)=s_{Y}(-i)=-i .
$$

Now, we show that the equality

$$
s_{X}(j)=s_{Y}(j)
$$

holds for every $j \neq \pm i$. Since $s_{X}$ and $s_{Y}$ are symplectic, it is sufficient to establish (3) only in the case when $j \notin X \cup Y$. Indeed, if $j \in X \cap Y$ then $-j$ does not belong to $X \cup Y$.

Let $j$ be an element of $\mathbb{Z} \backslash\{0\}$ which does not belong to $X \cup Y$. Then $-j \in X \cap Y$ and

$$
X^{\prime}:=\{j\} \cup(X \backslash\{-j\}) \in X^{\sim}, \quad Y^{\prime}:=\{j\} \cup(Y \backslash\{-j\}) \in Y^{\sim}
$$

are adjacent. Hence

$$
f\left(X^{\prime}\right)=s_{X}\left(X^{\prime}\right)=\left\{s_{X}(j)\right\} \cup\left(X \backslash\left\{-s_{X}(j)\right\}\right)
$$

and

$$
f\left(Y^{\prime}\right)=s_{Y}\left(Y^{\prime}\right)=\left\{s_{Y}(j)\right\} \cup\left(Y \backslash\left\{-s_{Y}(j)\right\}\right)
$$

are adjacent. The latter is possible only in the case when $s_{X}(j)=s_{Y}(j)$.

Using the connectedness of $H(A)$ and Lemma 4, we establish that $s_{X}=s_{Y}$ for all $X, Y \in H(A)$.

\section{Automorphisms of connected components}

Let $G_{1}$ and $G_{2}$ be permutation groups on sets $X_{1}$ and $X_{2}$, respectively. Recall that the wreath product $G_{1}<G_{2}$ is a permutation group on $X_{1} \times X_{2}$ and its elements are compositions of the following two types of permutations:

(1) for each element $g \in G_{2}$, the permutation $\left(x_{1}, x_{2}\right) \rightarrow\left(x_{1}, g\left(x_{2}\right)\right)$;

(2) for each function $i: X_{2} \rightarrow G_{1}$, the permutation $\left(x_{1}, x_{2}\right) \rightarrow\left(i\left(x_{2}\right) x_{1}, x_{2}\right)$.

Consider the subgroup of $G_{1} \prec G_{2}$ whose elements are compositions of permutations of type (1) and permutations of type (2) such that the set

$$
\left\{x_{2} \in X_{2}: i\left(x_{2}\right) \neq \operatorname{id}_{X_{1}}\right\}
$$

is finite. This is a proper subgroup only in the case when $X_{2}$ is infinite; it will be called the weak wreath product and denoted by $G_{1} 2_{w} G_{2}$. 
Corollary 5. The automorphism group of the connected component of $H_{\aleph_{0}}$ is isomorphic to the weak wreath product $S_{2} \imath_{w} S_{\aleph_{0}}$.

Proof. Let $A \in H_{\aleph_{0}}$ and $f$ be an automorphism of the connected component $H(A)$. By the previous section, $f$ is induced by a symplectic permutation $s$. Since $f(A)=s(A)$ belongs to $H(A)$, the set $s(A) \backslash A$ is finite. So, the automorphism group of $H(A)$ is isomorphic to the group of symplectic permutations $s$ such that the set $s(A) \backslash A$ is finite. The latter group is isomorphic to the weak wreath product $S_{2} \imath_{w} S_{\aleph_{0}}$ (indeed, we can identify the set $\mathbb{Z} \backslash\{0\}$ with the Cartesian product $\mathbb{Z}_{2} \times A$ and the group $S_{\aleph_{0}}$ with the group of all permutation on $A$ ).

\section{Acknowledgements}

I express my deep gratitude to Wilfried Imrich for useful information.

\section{References}

[1] A. Blunck, H. Havlicek. On bijections that preserve complementarity of subspaces. Discrete Math., 301:46-56, 2005.

[2] P. J. Cameron. Automorphisms of graphs. In Topics in Algebraic Graph Theory, volume 102 of Encyclopedia of Mathematics and Its Applications, pages 137-155. Cambridge University Press 2005.

[3] P. Erdős, A. Rényi. Asymmetric graphs. Acta Math. Acad. Sci. Hungar., 14:295-315, 1963.

[4] R. Frucht. Herstellung von Graphen mit vorgegebener abstrakter Gruppe. Compositio Math., 6:239-250, 1938.

[5] W. Imrich. Über das schwache Kartesische Produkt von Graphen. J. Combin. Theory Ser. B, 11:1-16, 1971.

[6] D. J. Miller. The automorphism group of a product of graphs. Proc. Amer. Math. Soc., 25:24-28, 1970.

[7] M. Pankov. Grassmannians of classical buildings. Algebra and Discrete Math. Series, no. 2. World Scientific, Singapore, 2010.

[8] M. Pankov. Automorphisms of infinite Johnson graphs. Discrete Math., accepted. 